\title{
Morbidade, suporte do parceiro e representações do tabaco em fumantes e abstinentes
}

\section{Morbidity, Partner Support and Tobacco Representations in Smokers and Abstinents}

\author{
Fernanda Afonso* \\ Universidade do Minho - Uminho, Braga, Portugal \\ Maria da Graça Pereira** \\ Universidade do Minho - Uminho, Braga, Portugal
}

\begin{abstract}
RESUMO
Este estudo comparou a morbidade psicológica, suporte do parceiro e representações face ao tabaco em 224 fumantes e 169 abstinentes. Os abstinentes, comparados com os fumantes, apresentaram mais suporte do parceiro, positivo e negativo, percecionaram mais consequências do tabaco, maior duração do seu consumo, menor controle pessoal, identificaram mais sintomas, maior preocupação e maior resposta emocional associada ao tabaco. Os fumantes apresentaram menos controle de tratamento. Nos fumantes e abstinentes verificaram-se várias relações significativas entre a morbidade psicológica, as representações face ao tabaco e o suporte do parceiro. Os preditores do suporte do parceiro, nos fumantes, foram o sexo, a idade, morbidade psicológica e controle do tratamento. Por sua vez, nos abstinentes, os preditores foram a idade, a ansiedade, a identidade e a resposta emocional.
\end{abstract}

Palavras-chave: Tabagismo, Morbidade, Representações, Suporte do Parceiro.

\section{ABSTRACT}

This study compared psychological morbidity, tobacco representations and partner support in 224 smokers and 169 abstinents. Abstinents when compared to smokers, revealed more partner support, positive and negative, more consequences of smoking, longer consumption of tobacco, less personal control, identity, more symptoms related to smoking, more concerns and emotional response associated to tobacco use. Smokers reported less treatment control. In both groups, smokers and abstinents, significant relationships were found between psychological morbidity, tobacco representations and partner support. Predictors of partner support, in smokers, were sex, age, psychological morbidity and treatment control. In abstinents the best predictors were age, anxiety, identity and emotional response.

Keywords: Smoking, Morbidity, Representations, Partner Support.

\section{I ntrodução}


A literatura mostra que o tabagismo assume um lugar de destaque entre os consumos de substâncias dado as suas consequências a nível físico e sintomatologia psicológica (MARTINET; BOHADANA, 2003). Segundo a Organização Mundial de Saúde, OMS, morrem atualmente em todo o mundo cerca de cinco milhões de pessoas devido a causas relacionadas com o tabagismo, o equivalente a um em cada dez indivíduos. Este número poderá ascender, em 2030, a cerca de dez milhões de pessoas por ano em todo o mundo (WORLD HEALTH ORGANIZATION, WHO, 2009). É relevante referir que o consumo do tabaco afeta, cada vez mais, populações dos países em desenvolvimento, onde se encontram atualmente $82 \%$ dos fumantes mundiais (cerca de 950 milhões de pessoas) (WORLD DEVELOPMENT REPORT, 2003). Atualmente, o consumo de tabaco é responsável pela morte de cerca de 5,4 milhões de pessoas por ano em todo o mundo, ou seja, uma em cada dez mortes ocorridas na idade adulta será atribuível ao consumo de tabaco (WHO, 2009). Em Portugal, segundo o mesmo estudo, a prevalência de consumo do tabaco situava-se nos $17,6 \%$ nos homens e de $26,2 \%$ nas mulheres, o que implica uma ligeira diminuição nos hábitos tabágicos no sexo masculino e um aumento de mais de $10 \%$ no sexo feminino, comparativamente com os anos de 1997/8 (FRAGA, SOUSA, SANTOS, MELLO, LUNET, PADRÃO, BARROS, 2005; LE FOLL; GEORGE, 2007). Dados do Inquérito Nacional de Saúde (INS), realizado entre 2005 e 2006, revelaram que $20,9 \%$ da população com 15 e mais anos de idade, residente em Portugal (incluindo as regiões autónomas) era fumante à data da entrevista (sexo masculino: $30,9 \%$; sexo feminino: $11,8 \%$ ) e que $18,7 \%$ fumava diariamente. Mais de metade dos homens fumava ou já tinha fumado $(56,9 \%)$, ao contrário das mulheres que, na grande maioria, nunca tinham fumado $(81,3 \%)$. As prevalências mais elevadas de homens que fumavam diariamente foram observadas nos Açores (31,0\%) e no Alentejo (29,9\%). Já entre as mulheres, a prevalência mais elevada de consumo diário de tabaco ocorreu na região de Lisboa e Vale do Tejo $(15,4 \%)$, seguida do Algarve $(12,8 \%)$. Em média, os homens fumavam mais cigarros por dia (20 cigarros) do que as mulheres (13 cigarros). A população açoriana evidenciou os consumos médios diários mais elevados (homens: 23 cigarros; mulheres: 16 cigarros).

Uma vez iniciado o consumo de tabaco, rapidamente se transforma num comportamento de risco altamente dependente (física e psiquicamente), sendo provocado principalmente pela nicotina (BECOÑA, 2003; NUNES, 2002). Este componente, só por si, provoca vários efeitos no corpo humano, nomeadamente a nível físico: aceleração do ritmo cardíaco, aumento do consumo de oxigênio pelo miocárdio, aumento da pressão sanguínea, aumento da capacidade de coagulação que pode levar á formação de coágulos e levar a 
colapsos (PESTANA, MENDES, 1999; PRECIOSO, VISEU, VILAÇA, HENRIQUES, LACERDA, 1991). Não só a nicotina é responsável pelos problemas de saúde, mas todo um conjunto de mais de 4000 componentes tóxicos que se encontram no fumo do tabaco dos quais 40 são de caráter carcinogênico como é o caso do monóxido de carbono, benzeno, formaldeído, chumbo, nitrosaminas, acetona, alcatrão, entre outras (NUNES, 2002; ROSAS, BAPTISTA, 2002)

O consumo da nicotina está associado a uma redução da ansiedade, do stress e da severidade da depressão (MACHAIN, VÉLEZ, GARCÍA, LUGO, GARCÍA, MORA, 2008). Verifica-se uma comorbidade entre o uso de substâncias (entre as quais o tabaco) e alterações de humor (MARTINEZ-ORTEGA, JURADO, GONZALEZ, GURPEGUI, 2006). De acordo com Malbergier e Oliveira J r. (2005) desde o fim da década de 70, passou a relacionar-se o tabaco com tensão, ansiedade, irritabilidade, e sintomas depressivos em geral. Há forte evidência de comorbidade entre tabagismo e transtornos depressivos (RONDINA, BOTELHO, GORAYEB, 2002) em que fumantes deprimidos fumam para aliviar os seus sintomas, reforçando ainda mais o desejo de fumar (GLASSMAN, COVEY, STETNER, RIVELLI, 2001). Estes fumantes embora conscientes do risco de fumar identificam o cigarro como uma fonte de prazer quando comparados com fumantes sem perturbações psicológicas. Estudos que focaram o impacto neurobiológico da nicotina no cérebro e a sua relação com a depressão, sublinham os fatores genéticos que podem ocorrer em mais de $67 \%$ dos indivíduos ao longo do processo de iniciação, manutenção e dependência de nicotina (PAPERWALLA, LEVIN, WEINER, SARAVAY, 2004). Estudos epidemiológicos, e clínicos, têm demonstrado uma associação positiva entre tabagismo e transtornos de ansiedade, ressaltando que a relação tabaco-ansiedade depende do tipo ou diagnóstico do distúrbio de ansiedade (RONDINA, BOTELHO, GORAYEB, 2002). De acordo com Malbergier e Oliveira Jr. (2005) a prevalência de tabagismo entre os indivíduos com transtornos de ansiedade $(47 \%)$ fica acima da prevalência na população geral. Do ponto de vista do comportamento tabágico, verifica-se que a nicotina pode atuar como um ansiolítico e um ansiogênico (HALLSTROM, MCCLURE, 1998). No que se refere à relação tabaco-ansiedade estudos revelam que fumantes com altos índices de ansiedade demonstram maiores dificuldades de adaptação no início de um método de cessação tabágica quando comparados com sujeitos com níveis baixos de morbidade psicológica em condições semelhantes (HALL, TUNSTALL, RUGG, JONES, BENOWITZ, 1985). Esta ansiedade é percecionada como incapacitante impelindo o indivíduo a fumar para diminuir ao seu estado de ansiedade (BARRETO, 1988). Os sintomas relacionados com a depressão também estão presentes no processo de abstinência tabágica e atingem o seu apogeu na primeira semana visto que estão associados a sintomas físicos e psicológicos 
(entre os quais o craving, i.e., sensação urgente de querer fumar) diminuindo nas semanas seguintes (GIOVINO, 1996). Fumantes com elevado stress psicológico referem mais dificuldades na cessação tabágica, sendo assim mais reduzida a probabilidade de se tornarem abstinentes (e.g., DUBE, CARABALLO, DHINGRA, PEARSON, MCCLAVE, STRINE, 2009). A recaída tende a ocorrer em períodos de stress e estados afetivos negativos, contribuindo para o aumento do stress psicológico (e.g., MANNING, CATLEY, HARRIS, MAYO, AHLUWALIA, 2005). Por sua vez, a ansiedade também pode estar presente no processo de cessação tabágica. Em um estudo realizado com 101 sujeitos fumantes integrados num programa de cessação tabágica verificou-se, após um mês de abstinência, que apresentavam um leve aumento dos índices de ansiedade (WEST, HAJEK, 1997). A ansiedade também está presente no momento de tomada de decisão para deixar de fumar, ou seja, o indivíduo pode sentir receio de não conseguir lidar com a ansiedade sem recurso ao tabaco (sensação de dificuldade em relação à ansiedade/helplessness e catastrofização). O follow-up controlado é importante na manutenção de abstinência para controle dos sintomas relacionados com a morbidade psicológica (BAER, MARLATT, 1991). O tabagismo está associado frequentemente a uma estratégia para lidar com situações stressantes (HITSMAN, BORRELLI, MCCHARGUE, SPRING, NIAURA, 2003; LENNOX, 1992; MAGID, CRAIG, COLDER, STROUD, NICHTER, 2008). Um estudo efetuado por Liu (2003), com uma amostra de 1360 adolescentes chineses, verificou que os fumantes mais velhos relataram terem vivenciado mais situações de stress face aos não-fumantes. O tabagismo foi associado a um maior risco para a internalização e problemas de comportamento, mas a associação foi significativamente reduzida após o controle de situações de stress, i.e. quanto mais controlado o stress, menos os adolescentes fumavam. No que respeita à abstinência tabágica também se verificam índices de stress. O stress desempenha um papel importante no tratamento da dependência de drogas, incluindo o tabagismo, podendo provocar recaídas em fumantes em fase de abstinência tabágica. Verificou-se que algumas regiões cerebrais préfrontais estavam relacionadas, após privação do cigarro, com o aumento dos níveis de stress que induziam respostas neuronais relacionadas com a vontade de fumar (DAGHER, TANNENBAUM, HAYASH, PRUESSNER, MCBRIDE, 2009). Assim o stress induz o uso do cigarro mesmo já em fase de abstinência tabágica.

Um outro aspeto presente nas diferentes fases do comportamento tabágico (iniciação, manutenção e cessação tabágica) é a influência do parceiro (PARK, TUDIVER, SCHULTZ, CAMPBELL, 2004; RÜGE, ULBRICHT, SCHUMANN, RUMPF, JOHN, MEYER, 2008). Este aspeto tem vindo a ser estudado como um fator-chave no fumante para lidar com situações de stress ajudando a mitigar estados de humor 
deprimido, ansiedade e estado geral de desânimo (DORMANN, ZAPF, 1999; ZELLARS, PERREWE, 2001). Desta forma, o parceiro pode influenciar a intenção do fumante para deixar de fumar (RÜGE, ULBRICHT, SCHUMANN, RUMPF, JOHN, MEYER, 2008) ou mesmo ter um papel decisivo na mudança de comportamento tabágico (HOMISH, KENNETH, 2005). Estudos revelam que o suporte recebido do parceiro é altamente preditivo do sucesso de uma desabituação tabágica em particular se a ajuda do companheiro for de índole cooperativa, tal como falar com o outro para não fumar e reforçar as condutas para deixar de fumar. Este suporte positivo pode ser preditor de sucesso e ser altamente funcional no que respeita a resultados efetivos e motivação do fumante ou abstinente. Afonso (2008) verificou nos fumantes, e nos abstinentes, uma relação positiva entre o suporte do parceiro e a dependência nicotínica indicando que maior dependência nicotínica estava associada a maior suporte do parceiro para deixar de fumar. Estes resultados evidenciam que o suporte do parceiro pode ser importante na mudança do comportamento tabágico (MERMELSTEIN, LICHTENSTEIN, MCINTYRE, 1983; ROHRBAUGH, SHOHAM, TROST, MURAMOTO, CATE, LEISCHOW, 2001). Ginsberg, Hall e Rosinsky (1991) observaram que o suporte positivo dado ao fumante, pelo companheiro, encoraja a sua autonomia e intenção para a mudança do comportamento tabágico. De fato, parceiros que não fumam, influenciam o fumante a deixar de fumar, enquanto os que fumam podem não ser tão encorajadores (DOBKIN, CIVITA, PARAHERAKIS, GILL, 2002). Ao nível da relação entre ajustamento conjugal e suporte do parceiro, Afonso (2008) não encontrou relações significativas em fumantes nem em abstinentes. Embora os estudos indiquem que quanto mais envolvidos os membros do casal estiverem na cessação tabágica mais eficaz é o suporte do parceiro (ROHRBAUGH, SHOHAM, TROST, MURAMOTO, CATE, LEISCHOW, 2001), pode acontecer que a dinâmica de casal não seja influenciada pelo consumo tabágico diretamente devido ao fato do casal, quando se conheceu, o parceiro já fumar ou o parceiro tem conhecimento da motivação do fumante para deixar de fumar.

Ao nível das representações do tabaco, muitos fumantes não reconhecem ter um problema de saúde, minimizam a importância da sua dependência, recusam a sua própria vulnerabilidade ou evocam exemplos de pessoas conhecidas que adoeceram sem nunca terem fumado (representações desajustadas acerca do problema) (MARTINET, BOHADANA, 2003; TRIGO, 2005). Fumar pode ser percebido como um comportamento com consequências irrelevantes para a saúde. De acordo com Afonso (2008) verifica-se, junto de fumantes, representações mais negativas face às consequências do comportamento tabágico. Assim, embora os fumantes continuem a fumar, apresentam uma perceção negativa das consequências do seu 
comportamento e dos riscos associados. Trigo (2005) apresenta um fenômeno cognitivo designado de "duplo nó psicológico" que se verifica em fumantes regulares em que a gratificação imediata pelo uso do cigarro é confrontada pela "escolha de um prazer que provoca dependência mortal". Assim, apesar de o uso do cigarro oferecer sensações de prazer e bem-estar, por outro lado existe a noção dos seus malefícios reais. No que respeita à abstinência, o tipo de representações é alterado assistindo-se a uma mudança de papéis em relação à passagem do estatuto de fumante, para ex-fumante, com uma visão mais realista dos malefícios do tabaco (BARRETO, 1988).

O presente trabalho teve por objetivo analisar: as diferenças entre fumantes e abstinentes ao nível da morbidade, suporte do parceiro e representações do tabaco; a relação entre morbidade (stress, depressão e ansiedade), representações do tabaco e suporte do parceiro e conhecer os preditores do suporte do parceiro em fumantes e abstinentes.

\section{Método}

\subsection{Participantes}

Para este estudo foram selecionados 224 fumantes $(52.7 \%$ sexo masculino, $47.3 \%$ sexo feminino) e 169 abstinentes, há pelo menos 3 meses, $(68.6 \%$ do sexo masculino e $31.4 \%$ do sexo feminino). Da amostra dos fumantes, $49.1 \%$ eram solteiros e $61.6 \%$ tinham o $12 \circ$ ano de escolaridade. $\mathrm{O}$ grupo mais numeroso pertenceu ao intervalo de idades (21-30 anos) com $41.1 \%$, logo seguido do intervalo ( $31-40$ anos) com $32.5 \%$. A maioria dos fumantes fumavam há mais de 3 anos e a idade mais frequente para começar a fumar situou-se entre 14 e os 18 anos. Dos fumantes, $60 \%$ efetuaram, pelo menos, uma tentativa para deixar de fumar mas sem sucesso. Cabe referir que $96 \%$ nunca frequentaram qualquer tratamento ou programa para deixar de fumar e $56.7 \%$ dos fumantes tinham um parceiro/a que não fumava.

Em relação aos abstinentes, $72.8 \%$ eram casados e $39 \%$ tinham o $12^{\circ}$ ano. O grupo mais numeroso $(27.2 \%)$ pertenceu ao intervalo de idades (41-50 anos) seguido do intervalo (31-40) com $20.1 \%$. A maioria dos inquiridos começou a fumar entre os 12 e os 18 anos. Todos os sujeitos efetuaram pelo menos uma tentativa para deixar de fumar sendo que $32 \%$ tinham procurado tratamento para deixar de fumar.

\subsection{Procedimentos de coleta de dados}


Os participantes foram recrutados num hospital central, numa empresa privada e numa universidade no norte de Portugal. Os participantes foram selecionados a partir de uma reunião geral, em cada local, em que foram apresentados os objetivos do estudo. A participação dos sujeitos foi voluntária sendo o seu consentimento de resposta precedido de informação sobre o âmbito e finalidade do estudo. O preenchimento dos instrumentos efetuou-se num momento único e todos os participantes assinaram o Termo de Consentimento Livre e Esclarecido(TCLE). Os critérios de inclusão para a amostra foram: ser maior de 18 anos, ser fumante diário, e no caso dos abstinentes, estar sem fumar há pelo menos 3 meses.

\subsection{Procedimentos éticos}

Sem conflito de interesse, a pesquisa foi aprovada pelo Comitê de Ética de cada local de recolha dos dados recebendo aprovação: universidade (protocolo $\mathrm{n}$ 536807), empresa (protocolo $\mathrm{s} / \mathrm{n}$ 으) e hospital (protocolo $\mathrm{s} / \mathrm{n}$ 으) .

\subsection{I nstrumentos}

- Questionário Sócio-Demográfico (PEREIRA \& AFONSO, 2009) tinha como objetivo recolher informações para caracterizar os sujeitos da amostra ao nível de: estado civil, sexo, habilitações literárias, tipo e quantidade de cigarros consumidos (fumante), duração da abstinência (abstinente).

- Brief Illness Perception Questionnaire (IPQ-Brief) (BROADBENT, PETRIE, MAIN, \& WEINMAN, 2006; versão portuguesa de FIGUEIRAS, MARCELINO, CLAUDINO, CORTES, MAROCO, WEINMAN, 2010). Este instrumento é constituído por nove itens que permitem avaliar as representações emocionais e cognitivas da doença. O IPQ-breve utiliza uma escala LIKERT em que todos os itens, com exceção da questão 9 , relativa à Causa, são respondidos numa escala de zero a dez. Os itens organizam-se em: Consequências (item 1), Duração (item 2), Controle Pessoal (item 3), Controle através do Tratamento (item 4) e Identidade (item 5). Um item avalia a Compreensão da doença (item 7). A avaliação das representações causais é feita através de uma questão aberta adaptada do IPQ-R, que questiona 0 paciente acerca das três causas mais importantes para a sua doença (item 9), mas que não foi incluída neste estudo. Dado que as subescalas do instrumento são constituídas por um item não permite efetuar estudos de fidelidade ou validade. De referir que no questionário se substituiu o termo "doença" por "consumo tabágico" para os fumantes, e por "a minha vontade de fumar" para os abstinentes para efeitos da investigação. Os itens 3, 4 e 7 são 
invertidos. Um valor elevado em cada item representa maior intensidade no tipo de crença avaliada.

- Partner Interaction Questionnaire (COHEN \& LICHTENSTEIN, 1990, versão de investigação de PEREIRA \& AFONSO, 2006). Este instrumento é constituído por uma escala positiva ( 8 itens, alfa $=.73$ ) e uma escala negativa (11 itens, alfa $=.84$ ), que avaliam o apoio recebido, nos últimos três meses para deixar de fumar. Foi utilizada uma versão adaptada para fumantes e abstinentes. Um resultado elevado significa maior apoio, positivo ou negativo, por parte do parceiro. O apoio positivo refere-se ao reforço positivo do parceiro para que o fumante deixe de fumar e o apoio negativo refere-se a uma atitude de indiferença, ou de reforço negativo, por parte do parceiro, para deixar de fumar.

- Escala de Ansiedade, Depressão e Stress (EADS) (LOVIBOND \& LOVIBOND, 1995; versão portuguesa de PAIS-RIBEIRO, HONRADO, LEAL, 2004). Este instrumento é constituído por 21 itens organizados em três subescalas: Ansiedade ( 7 itens), Depressão ( 7 itens) e Stress (7itens). Na nossa amostra os valores de alfa foram de .71, .83 e .83 , respetivamente. A EADS fornece três pontuações, uma para cada subescala (Depressão, Ansiedade e Stress), em que a mínima é "0" e a máxima "21". Para se obter o resultado de cada uma das subescalas somam-se os valores dos sete itens correspondentes. Quanto mais elevada a pontuação, maior o índice de morbidade psicológica.

\subsection{Análise de dados}

Com o objetivo de averiguar a existência de diferenças entre a morbidade psicológica (depressão, ansiedade e stress), representações do tabaco e suporte do parceiro (positivo e negativo) entre os grupos, recorreu-se ao uso da Mancova. Foram controladas as variáveis idade, habilitações literárias e sexo dado os grupos apresentarem diferenças nestas variáveis. Para análise da relação entre a morbidade psicológica, suporte do parceiro e representações do tabaco foi utilizado o teste de correlação de Pearson. Ao nível da predição do suporte do parceiro foi efetuada uma regressão linear (método enter).

\section{Resultados}

1. Diferenças entre Fumantes e Abstinentes ao nível da Morbidade Psicológica, Suporte do Parceiro e Representações do Tabaco Verificaram-se diferenças significativas, entre fumantes e abstinentes, ao nível da ansiedade indicando que os abstinentes apresentaram níveis mais elevados de ansiedade quando comparados 
com os fumantes. Ao nível do suporte do parceiro, verificou-se que os abstinentes relataram mais suporte positivo e negativo, por parte do parceiro, indicando ter recebido mais suporte para deixar de fumar. Ao nível das representações associadas ao tabaco verificou-se, no grupo dos abstinentes, mais consequências associadas ao uso do tabaco, maior duração do hábito de fumar, mais controle pessoal, mais identidade, mais preocupações e resposta emocional associada ao tabaco. No grupo dos fumantes, verificaram-se diferenças ao nível do controle de tratamento indicando que os fumantes acreditavam que o tratamento para o tabaco não iria ser eficaz para deixar de fumar conforme apareceu na Tabela1:

\begin{tabular}{|c|c|c|c|c|}
\hline & Variáveis & $\begin{array}{l}\text { Fumadores } \\
(N=224) \\
M(D P)\end{array}$ & $\begin{array}{l}\text { Abstinentes } \\
\text { (N=169) } \\
M(D P)\end{array}$ & $\mathrm{F}(1,391)$ \\
\hline \multirow{3}{*}{$\begin{array}{l}E \\
A \\
D \\
S\end{array}$} & Depressão & $3.044(3.671)$ & $3.378(4.221)$ & .701 \\
\hline & Ansiedade & $2.379(1.994)$ & $3.284(3.748)$ & $9.491 * *$ \\
\hline & Stress & $3.848(2.608)$ & $3.982(3.747)$ & .175 \\
\hline \multirow{2}{*}{$\begin{array}{l}\mathrm{P} \\
\mathrm{I} \\
\mathrm{Q}\end{array}$} & Escala_Positiva & $16.660(5.688)$ & $19.958(6.458)$ & $28.796 * * *$ \\
\hline & Escala_Negativa & $14.861(7.995)$ & $22.100(9.223)$ & $69.130 * * *$ \\
\hline \multirow{8}{*}{$\begin{array}{l}\mathrm{B} \\
- \\
\mathrm{I} \\
\mathrm{P} \\
\mathrm{Q}\end{array}$} & Consequências & $3.852(2.670)$ & $4.887(3.280)$ & $11.824 * *$ \\
\hline & Duração & $4.821(2.832)$ & $7.268(3.184)$ & $64.357 * * *$ \\
\hline & Controlo Pessoal & $3.335(2.71)$ & $5.342(3.289)$ & $43.864 * * *$ \\
\hline & Controlo Tratamento & $6.817(3.284)$ & $5.405(4.335)$ & $13.468 * * *$ \\
\hline & Identidade & $3.080(2.349)$ & $5.017(3.176)$ & $48.207 * * *$ \\
\hline & Preocupação & $5.018(3.157)$ & $5.982(3.245)$ & $8.745^{* *}$ \\
\hline & Compreensão & $3.607(2.711)$ & $3.881(3.350)$ & .797 \\
\hline & Resposta emocional & $3.438(2.980)$ & $4.958(3.053)$ & 24.519*** \\
\hline \multicolumn{5}{|c|}{$* * * p \leq .000 ; * * p \leq .001$} \\
\hline
\end{tabular}

2. Relação entre Morbidade Psicológica, Suporte do Parceiro e Representações do Tabaco em Fumantes e Abstinentes.

No grupo dos fumantes verificou-se uma relação positiva entre a morbidade psicológica (depressão, ansiedade e stress) e as representações associadas ao tabaco (consequências associadas ao tabaco, controle pessoal, identidade, preocupação, compreensão e resposta emocional) (Tabela 2 ). Estes resultados sugeriram que representações negativas associadas ao hábito de fumar estavam associadas a sintomas de morbidade psicológica. Verificou-se uma relação positiva entre o suporte positivo do parceiro e o controle do tratamento. Verificou-se, também, uma correlação negativa entre a morbidade psicológica e o suporte positivo do parceiro, entre a morbidade psicológica e as representações associadas ao tabaco (duração e o controle do tratamento). Os resultados revelaram uma relação negativa entre as consequências e o controle do tratamento e entre as consequências e compreensão; entre a duração e controle 
pessoal, e entre a duração e preocupação conforme se verifica na Tabela 2.

No grupo dos abstinentes verificou-se uma relação positiva ao nível das representações, nomeadamente, entre as consequências e: a duração, o controle pessoal, a identidade, a preocupação e a resposta emocional. Também se verificou uma relação negativa entre a morbidade psicológica e a compreensão do hábito de fumar, entre a morbidade psicológica e o suporte negativo do parceiro e entre a morbidade psicológica e o controle de tratamento, bem como uma relação negativa entre a preocupação associada ao hábito de fumar e a identidade, e entre a resposta emocional e o controle do tratamento conforme se verificou na Tabela 2:

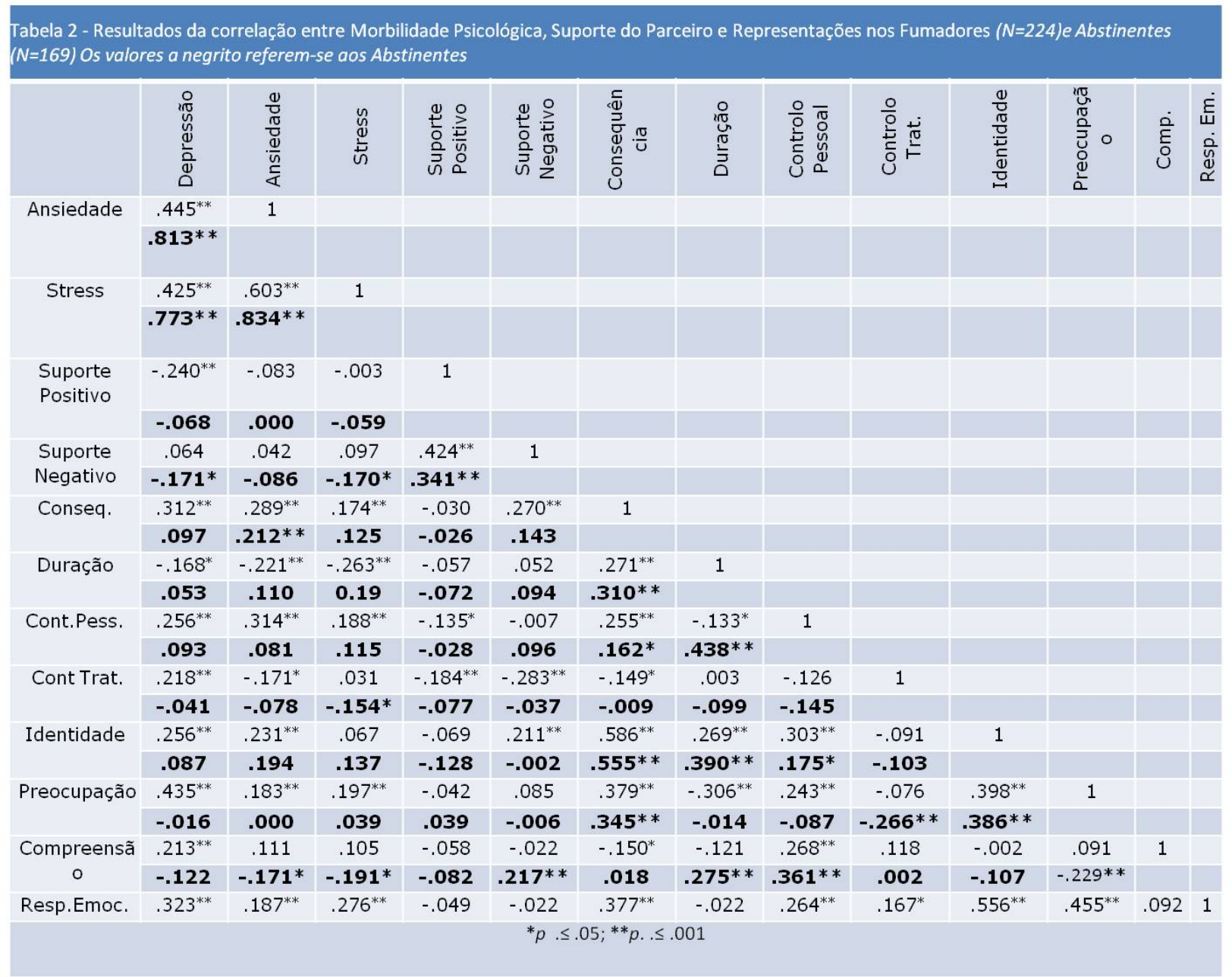

3. Preditores do Suporte do Parceiro em Abstinentes e Fumantes.

Nos fumantes, o sexo e a idade foram preditores de mais suporte positivo por parte do parceiro. Ao nível das variáveis psicológicas, a morbidade psicológica (depressão e stress) e controle do tratamento mostraram ser preditores de mais suporte positivo do parceiro enquanto a ansiedade, stress, consequências, controle do tratamento 
e identidade mostraram ser preditores de mais suporte negativo conforme se verificou na Tabela 3:

Tabela 3- Preditores do Suporte do Parceiro nos Fumadores $(N=224)$

\begin{tabular}{|c|c|c|c|c|c|c|c|c|}
\hline \multirow[t]{3}{*}{ Variáveis } & \multicolumn{8}{|c|}{ Preditores } \\
\hline & \multicolumn{4}{|c|}{ Suporte Positivo } & \multicolumn{4}{|c|}{ Suporte Negativo } \\
\hline & $R^{2}$ & B & SE B & $\beta$ & R2 & B & SE B & $\beta$ \\
\hline Bloco 1 & .091 & & & & 107 & & & \\
\hline Sexo & & 1.406 & .725 & .126 & & 4.421 & 1.064 & $.268 * * *$ \\
\hline Idade & & -1.390 & .425 & $-.232^{* *}$ & & -2.244 & .624 & $-.253 * * *$ \\
\hline $\begin{array}{l}\text { Duração do } \\
\text { consumo }\end{array}$ & & -.905 & .674 & -.095 & & 1.396 & .988 & .099 \\
\hline \multicolumn{5}{|c|}{$\mathbf{R}^{2} \mathbf{a d j} .: .078$} & \multicolumn{4}{|c|}{$\mathbf{R}^{2}$ adj . :.095 } \\
\hline Bloco 2 & .202 & & & & .283 & & & \\
\hline Sexo & & 1.726 & .750 & $.155^{*}$ & 3.637 & 1.052 & .220 & $3.637 * *$ \\
\hline Idade & & -1.493 & .443 & $-.249 *$ & -2.029 & .621 & -.229 & $-2.029 * *$ \\
\hline $\begin{array}{l}\text { Duração do } \\
\text { consumo }\end{array}$ & & -.956 & .706 & -.100 & 1.064 & .991 & .075 & 1.064 \\
\hline Depressão & & -.434 & .138 & $-.268 *$ & .216 & .193 & .090 & .216 \\
\hline Ansiedade & & -.208 & .255 & -.074 & -1.015 & .358 & -.245 & $-1.015 * *$ \\
\hline Stress & & .449 & .184 & $.210 *$ & .890 & .258 & .281 & $.890 * *$ \\
\hline Consequências & & .087 & .189 & .042 & .604 & .265 & .195 & $.604 *$ \\
\hline Duração & & -.100 & .166 & -.051 & -.234 & .233 & -.080 & -.234 \\
\hline $\begin{array}{l}\text { Controlo } \\
\text { Pessoal }\end{array}$ & & -.119 & .157 & -.058 & -.344 & .220 & -.113 & -.344 \\
\hline $\begin{array}{l}\text { Controlo } \\
\text { Tratamento }\end{array}$ & & -.262 & .123 & $-.154^{*}$ & -.684 & .173 & -.272 & $-.684 * * *$ \\
\hline Identidade & & -.142 & .228 & .809 & .319 & .230 & .230 & $.809 * *$ \\
\hline Preocupação & & -.040 & .157 & -.216 & .221 & -.083 & -.083 & -.216 \\
\hline Compreensão & & .116 & .141 & .253 & .198 & .083 & .083 & .253 \\
\hline \multirow[t]{2}{*}{$\begin{array}{l}\text { Resposta } \\
\text { Emocional }\end{array}$} & & .260 & .164 & -.324 & .230 & -.117 & -.117 & -.324 \\
\hline & \multicolumn{4}{|c|}{$\mathbf{R}^{2} \mathbf{a d j} .: .148$} & \multicolumn{4}{|c|}{$\mathbf{R}^{2} \mathbf{a d j} .: .235$} \\
\hline \multicolumn{5}{|c|}{$* * p . \leq .05 ; * * * p . . \leq .001$} & & & & \\
\hline
\end{tabular}

No grupo dos abstinentes, ao nível das variáveis demográficas, a idade foi preditora de mais suporte positivo e negativo do parceiro. A ansiedade, a identidade e a resposta emocional associada ao tabaco, ao nível das variáveis psicológicas foram preditoras de mais suporte positivo conforme se verificou na Tabela 4: 
Fernanda Afonso, Maria da Graça Pereira Morbidade, suporte do parceiro e representações do tabaco em fumantes e abstinentes

\begin{tabular}{|c|c|c|c|c|c|c|c|c|}
\hline \multirow[t]{3}{*}{ Variáveis } & \multicolumn{8}{|c|}{ Preditores } \\
\hline & \multicolumn{4}{|c|}{ Suporte Positivo } & \multicolumn{4}{|c|}{ Suporte Negativo } \\
\hline & $\mathrm{R}^{2}$ & B & SE B & $\beta$ & $\mathrm{R}^{2}$ & $B$ & SE B & $\beta$ \\
\hline Bloco 1 & .030 & & & & .123 & & & \\
\hline Sexo & & .576 & .990 & .049 & & 2.203 & 1.785 & .099 \\
\hline Idade & & -.738 & .338 & $-.190 *$ & & 1.814 & .609 & $.246 *$ \\
\hline \multirow{2}{*}{$\begin{array}{l}\text { Duração do } \\
\text { consumo }\end{array}$} & & .418 & .414 & .080 & & 1.260 & .747 & .127 \\
\hline & \multicolumn{4}{|c|}{$\mathbf{R}^{2}$ adj $\ldots .013$} & & & \multicolumn{2}{|c|}{$\mathbf{R}^{2}$ adj.:.107 } \\
\hline Bloco 2 & .153 & & & & .194 & & & \\
\hline Sexo & & -.062 & 1.040 & -.005 & & .851 & 1.923 & .038 \\
\hline Idade & & -.786 & .361 & $-.202 *$ & & 1.455 & .668 & $.198^{*}$ \\
\hline $\begin{array}{l}\text { Duração do } \\
\text { consumo }\end{array}$ & & .504 & .431 & .097 & & 1.355 & .796 & .137 \\
\hline Depressão & & -.288 & .195 & -.206 & & -.377 & .361 & -.142 \\
\hline Ansiedade & & .513 & .249 & $.355^{*}$ & & .401 & .460 & .146 \\
\hline Stress & & -.354 & .219 & -.245 & & -.367 & .405 & -.134 \\
\hline Consequências & & .024 & .169 & .015 & & .339 & .313 & .108 \\
\hline Duração & & -.036 & .160 & -.021 & & -.057 & .296 & -.018 \\
\hline $\begin{array}{l}\text { Controlo } \\
\text { Pessoal }\end{array}$ & & .170 & .153 & .103 & & -.032 & .283 & -.010 \\
\hline $\begin{array}{l}\text { Controlo } \\
\text { Tratamento }\end{array}$ & & -.083 & .106 & -.066 & & -.186 & .196 & -.078 \\
\hline Identidade & & -.448 & .173 & $-.262^{*}$ & & -.447 & .320 & -.138 \\
\hline Preocupação & & -.048 & .163 & -.028 & & -.195 & .301 & -.061 \\
\hline Compreensão & & -.120 & .144 & -.074 & & .384 & .266 & .125 \\
\hline \multirow[t]{2}{*}{$\begin{array}{l}\text { Resposta } \\
\text { Emocional }\end{array}$} & & .492 & .172 & $.276 * *$ & & .530 & .318 & .157 \\
\hline & \multicolumn{4}{|c|}{$\mathbf{R}^{2}$ adj . . ..075 } & \multicolumn{4}{|c|}{$\mathbf{R}^{2}$ adj... .121} \\
\hline$* * p . \leq .05 ; * *$ & $<$ & & & & & & & \\
\hline
\end{tabular}




\section{Discussão}

De acordo com os resultados, verificaram-se diferenças entre fumantes e abstinentes, ao nível da ansiedade indicando que os abstinentes apresentaram níveis mais elevados de ansiedade quando comparados com os fumantes. De fato, no processo de consumo e cessação tabágica, existe a probabilidade de desenvolver sintomatologia ansiosa (TSOH, HUMFLEET, MUÑOZ, REUS, HARTZ, HALL, 2000). A abstinência pode ser influenciada, ou facilmente quebrada, em indivíduos com maior morbidade psicológica, pois apresentam recaídas mais frequentes e mais rápidas quando comparados com sujeitos com índices inferiores de morbidade psicológica (GLASSMAN, COVEY, STETNER, RIVELLI, 2001). No que respeita à ansiedade, em particular, os sintomas ansiosos atingem o seu apogeu na primeira semana visto estarem associados a sintomas físicos e psicológicos (entre os quais o craving) diminuindo nas semanas seguintes (GIOVINO, 1996). Os fumantes com mais ansiedade, ou mais stress, referem mais dificuldades na cessação tabágica, sendo assim mais reduzida a probabilidade de se tornarem abstinentes (e.g., DUBE, CARABALLO, DHINGRA, PEARSON, MCCLAVE, STRINE, 2009). O aumento da ansiedade, no tratamento das dependências (e.g. o tabagismo) é um fator importante na recaída. Assim, a ansiedade sentida pelos abstinentes pode levar ao uso do cigarro (DAGHER, TANNENBAUM, HAYASH, PRUESSNER, MCBRIDE, 2009). A recaída tende a ocorrer em períodos de stress e estados afetivos negativos, contribuindo para o aumento do stress psicológico, ansiedade e depressão (e.g., MANNING, CATLEY, HARRIS, MAYO, AHLUWALIA, 2005; WEST, HAJEK, 1997). Ao nível do suporte do parceiro para deixar de fumar os abstinentes referem ter recebido mais suporte, positivo e negativo. A literatura salienta a importância do suporte do parceiro nas diferentes fases do comportamento tabágico em particular na cessação tabágica (PARK, TUDIVER, SCHULTZ, CAMPBELL, 2004; RÜGE, ULBRICHT, SCHUMANN, RUMPF, JOHN, MEYER, 2008).

Ao nível das representações associadas ao tabaco, os fumantes, apresentaram menos controle de tratamento o que sugere que consideram que o tratamento para deixar de fumar pode não ser eficaz. Podemos compreender estes resultados tendo em conta o modelo de Autorregulação de Leventhal, que refere que as pessoas com maior capacidade de resolução de problemas são aquelas que constroem representações cognitivas mais coerentes em relação ao seu estado de saúde (LEVENTHAL, CAMERON, 1987). Uma crença desajustada acerca de uma doença pode ajudar a construir uma estratégia de coping igualmente desajustada, o que pode afetar respostas emocionais e, eventualmente, causar uma avaliação 
imperfeita promotora de representações desajustadas. Assim, o fumante ao considerar que o tratamento para deixar de fumar pode não ser eficaz coloca em causa a sua saúde, continuando a fumar sem restrições. Os abstinentes apresentaram mais representações negativas associadas com o seu hábito de fumar revelando mais consequências associadas ao tabaco, mais duração do seu consumo de tabaco, menos controle quando fumavam, mais sintomas relacionados com fumar, mais preocupação com o seu hábito e mais sintomas emocionais negativos enquanto fumantes (PUSCHEL, THOMPSON, CORONADO, RIVERA, DIAZ, GONZALEZ, 2006).

No grupo dos fumantes, verificou-se uma relação positiva entre a morbidade psicológica (depressão, ansiedade e stress) e as representações associadas ao tabaco (consequências associadas ao tabaco, controle pessoal, identidade, preocupação, compreensão e resposta emocional). Estes resultados sugerem que o fumante, ao ter noção real das consequências que o tabagismo pode acarretar para a sua saúde, menos controle pessoal sobre fumar, ao aceitar que os sintomas relacionados com o seu hábito o preocupam, ao ter dificuldade em compreender o seu hábito e considerando que se sente afetado emocionalmente por fumar, pode apresentar mais índices significativos de depressão, ansiedade e stress (AFONSO, 2008; COLLINS, MOIRA, O'DELL, 2002). A relação positiva entre o suporte negativo do parceiro e as consequências associadas ao tabaco revela que os fumantes que têm mais suporte negativo do parceiro consideram que o consumo de tabaco afeta mais a sua vida. Estes resultados vão no sentido de quem tem mais apoio negativo do parceiro identifica que o hábito consumo de tabaco afeta a sua vida, e sente-se mais pressionado, pelo companheiro, para deixar de fumar (PARK, TUDIVER, SCHULTZ, CAMPBELL, 2004). A relação negativa entre a morbidade psicológica e o suporte positivo do parceiro indicou que mais suporte positivo, menor o índice de sintomas de depressão do fumante. Estes resultados podem ser explicados pelo fato de 0 apoio dado ao fumante para deixar de fumar ser bastante importante na mudança do comportamento tabágico e contribuir para a diminuição dos sintomas depressivos associados à adaptação à mudança (MERMELSTEIN, LICHTENSTEIN, MCINTYRE, 1983; ROHRBAUGH, SHOHAM, TROST, MURAMOTO, CATE, LEISCHOW, 2001). A relação negativa entre e a morbidade psicológica e a duração e o controle do tratamento pode indicar que os sintomas de morbidade diminuem à medida que o consumo de tabaco dura mais ou que o tratamento para deixar de fumar é considerado como podendo não ser eficaz. Desta forma, a gratificação imediata obtida ao fumar é confrontada pela escolha de um prazer com contornos nocivos para a saúde. É verificado, também que à medida que fumar afecta a vida do fumante (consequências) menor a percepção da eficácia do tratamento e menor a compreensão do seu consumo de 
tabaco. Quanto mais tempo o fumante fumar menor a perceção de controle sobre o seu hábito e menos compreende por que fuma (TRIGO, 2005).

No grupo dos abstinentes verificou-se uma relação positiva ao nível das representações, nomeadamente, entre as consequências e a duração, o controle pessoal, a identidade, a preocupação e a resposta emocional. Estes resultados indicam que quanto mais longo 0 consumo de tabaco dos abstinentes, quando eram fumantes, mais controle sentiam sobre fumar, mais identificavam sintomas relacionados com o consumo tabágico e mais se preocupavam, mais sentiam que fumar os afectava emocionalmente a sua vida (consequências). Por sua vez, os sintomas relacionados com a morbidade psicológica relacionaram-se inversamente com a compreensão do hábito de fumar, suporte negativo do parceiro e controle de tratamento. Assim, os abstinentes quando fumavam tinham mais dificuldades em compreender porque fumavam, recebiam menos apoio negativo do parceiro e consideravam que o tratamento para deixar de fumar poderia não ser eficaz e, por isso se compreende que estes dados estejam associados a sintomas de ansiedade e stress. Desta forma a mudança de comportamento poderia estar dificultada pela fraca gestão da morbidade psicológica (e.g., DUBE, CARABALLO, DHINGRA, PEARSON, MCCLAVE, STRINE, 2009). Os abstinentes, quando fumavam, à medida que estavam preocupados porque fumavam não apresentavam sintomas. Por sua vez, à medida que se sentiam afetados por fumarem consideravam que o tratamento para deixar de fumar não era eficaz e a cessação tabágica vista como um processo difícil (AFONSO, 2008).

No estudo dos preditores, nos fumantes, o sexo e a idade, a morbidade psicológica (depressão e stress), e controle do tratamento foram preditores do suporte positivo e negativo, por parte do parceiro. Nesse sentido, fumantes mais velhos, e do sexo feminino, tendem a receber mais apoio positivo e menos suporte negativo do parceiro, provavelmente pelo fato de que os parceiros consideram importante a mudança de comportamento, devido a problemas de saúde.

A depressão o stress e o controle do tratamento foram preditores de mais suporte positivo por parte do parceiro. Assim, ter mais sintomas depressivos e de stress, bem como considerar que o tratamento pode não ser eficaz para deixar de fumar (controle do tratamento) podem levar a que o parceiro forneça apoio positivo para deixar de fumar e promover uma vida mais saudável ao fumante (TSOH, HUMFLEET, MUÑOZ, REUS, HARTZ, HALL, 2000).

A ansiedade, o stress, consequências associadas ao tabaco, controle do tratamento e identidade mostraram ser preditores de suporte negativo dado pelo parceiro. $O$ fato de os fumantes perceberem que 0 tabaco é nefasto para a sua saúde, afecta a sua vida e a presenças 
de sintomas relacionados com o fato de fumar bem como sintomas de morbidade psicológica pode levar o parceiro a fornecer mais apoio negativo i.e. pressionar e criticar para que o fumante deixe de fumar (AFONSO, 2008; MERMELSTEIN; LICHTENSTEIN; MCINTYRE, 1983; PARK, TUDIVER, SCHULTZ, CAMPBELL, 2004; ROHRBAUGH, SHOHAM, TROST, MURAMOTO, CATE, LEISCHOW, 2001). Este apoio negativo não se caracteriza por ser totalmente desmotivador, pelo contrário, é um apoio em que o parceiro vigia e insiste para que o parceiro deixe de fumar (MERMELSTEIN; LICHTENSTEIN; MCINTYRE, 1983; DORMANN, ZAPF, 1999; VAN DIERENDONCK, SCHAUFELI, BUUNK, 2001; ZELLARS, PERREWE, 2001).

No grupo dos abstinentes, a idade foi preditora de mais suporte positivo dado pelo parceiro, o que pode indicar que os abstinentes mais velhos, quando eram fumantes, receberam mais apoio positivo por parte do parceiro para deixar de fumar. Isto pode se explicado pelo fato de os parceiros considerarem que depois de muito tempo a fumar existe a probabilidade de desenvolver problemas de saúde relacionados com o tabaco. A ansiedade, a identidade e a resposta emocional associada ao tabaco, no domínio das variáveis psicológicas, mostram ser preditoras de mais suporte positivo, i.e., quanto mais ansiedade resultante de fumar o abstinente apresentava, mais o parceiro promovia apoio no sentido da mudança de comportamento (ROHRBAUGH, SHOHAM, TROST, MURAMOTO, CATE, LEISCHOW, 2001). A identidade e a resposta emocional foram preditores de mais suporte positivo do parceiro dado que os abstinentes, quando fumavam, tinham noção dos sintomas relacionados com o tabaco, bem como o quanto fumar os afectava emocionalmente. Desta forma o apoio do parceiro foi um fator importante na mudança do comportamento.

\section{Limitações}

O fato de que a amostra foi de conveniência, recolhida apenas na zona norte de Portugal limita a generalização dos resultados. Desta forma, os resultados estão limitados a uma zona geográfica do país. A aplicação de medidas de autorrelato é também uma limitação devido à desejabilidade social das respostas dos participantes, i.e., as respostas dadas podem ser as que os participantes consideram mais adequadas ou as que seriam esperadas. Os abstinentes responderam aos instrumentos de forma retrospectiva em relação ao seu comportamento de fumar. Este aspeto pode sugerir que algumas dimensões avaliadas podem já estar pouco presentes e a resposta não corresponder à realidade. Finalmente, o comportamento de abstinência inclui uma duração igual/superior a três meses, pelo que seria importante, em estudos futuros, verificar se recém-abstinentes 
se distinguem de abstinentes de longa duração, nas variáveis estudadas.

\section{Conclusão}

Os resultados deste estudo salientam a importância de incluir nos programas de cessação tabágica as representações do tabaco. Estes resultados vão ao encontro da literatura que sublinha a importância das representações associadas ao tabaco (Trigo, 2005). Assim, se os fumantes perceberem que o consumo de tabaco tem consequências negativas para a saúde, e que o consumo se associa a morbidade psicológica, poderão sentir-se mais motivados para deixar de fumar, i.e., a perceção das consequências para a saúde, do ato de fumar, apesar das sensações positivas associadas (prazer e relaxamento) está associada a mais motivação para a mudança (Afonso, 2008).

Um dos contributos importantes deste estudo tem a ver com a importância do suporte do parceiro para deixar de fumar. Este tipo de suporte, quer positivo ou negativo, revela-se uma fonte de apoio fundamental para o fumante deixar de fumar (AFONSO, 2008; MERMELSTEIN; LICHTENSTEIN; MCINTYRE, 1983; PARK et al., 2004; ROHRBAUGH, SHOHAM, TROST, MURAMOTO, CATE, LEISCHOW, 2001). Neste sentido, do ponto de vista teórico, parece ser importante incluir nos modelos de mudança de comportamento tabágico esta variável bem como outras variáveis maritais que possam ser importantes como por exemplo, o valor que o parceiro atribui à saúde.

Este estudo destaca a importância de incluir nos programas de cessação tabágica o parceiro, as representações do tabaco e a diminuição da morbidade psicológica.

\section{Referências}

AFONSO, M. F. Variáveis individuais e familiares no comportamento tabágico. 2008. 181f. Dissertação de Mestrado (Mestrado em Psicologia da Saúde) - Universidade do Minho, Braga, Portugal.

BAER, J.S.; MARLATT, G.A. Maintenance of smoking cessation. Clinics in Chest Medicine, Philadelphia, v. 12, n.4, p. 793-800, 1991.

BARRETO, J. A desabituação do fumante. O Médico, p. 225-282, 1988.

BECOÑA, E. El Tratamiento Psicológico de la Adicción a la Nicotina. Papeles del Psicologo, Madrid, v. 85, n. 85, p. 48-69, 2003. 
BROADBENT, E.; PETRIE, K. J.; MAIN, J.; WEINMAN, J. The brief illness perception questionnaire. Journal of Psychosomatic Research, Manchester, v. 60, n. 6, p. 631-637, 2006.

COHEN, S.; LICHTENSTEIN, E. Partner Behaviours that support quitting smoking. J ournal of Consulting and Clinical Psychology, Washington, v. 58, n. 3, p. 304-309, 1990.

COLLINS, P.; MOIRA, M.; O'DELL, L. Smoker's Representations of their own smoking: a Q-methodological study. Journal of Health Psychology, Londres, v. 7, n. 6, p. 641-652, 2002.

DAGHER, A.; TANNENBAUM, T.; HAYASH, T; PRUESSNER, J.; MCBRIDE, D. An acute psychosocial stress enhances the neural response to smoking cues. Brain Research, Filadelfia, v.1293, n. 1, p. 40-48, 2009.

DOBKIN, P. L.; CIVITA, M. D.; PARAHERAKIS, A.; GILL, K. The Role of Functional Social Support in Treatment Retention and Outcomes among Outpatient Adult Substance Abusers. Addiction, Londres, v. 97, p. 347-356, 2002.

DORMANN, C.; ZAPF, D. Social Support, Social Stressors at Work, and Depressive Symptoms: Testing for Main and Moderating Effects with Structural Equations in a Three-Wave Longitudinal Study. Journal of Applied Psychology, Washington, v. 84, n.6, p. 874884, 1999.

DUBE, S. R.; CARABALLO, R. S.; DHINGRA, S. S.; PEARSON, W. S.; MCCLAVE, A. K.; STRINE, T. W. The relationship between smoking status and serious psychological distress: findings from the 2007 Behavioral Risk Factor Surveillance System. I nternational J ournal of Public Health, Basel,v. 54,n. 1, p. 68-74, 2009.

Figueiras, M.; Marcelino, D.; Claudino, A.; Cortes, M.; Maroco, J; Weinman, J. Patients' illness schemata of hypertension: the role of beliefs for the choice of treatment. Psychology \& Health, Oxford, v. 25, n. 4, p.507 - 517. 2010.

FRAGA S.; SOUSA S.; SANTOS A.C.; MELLO, M.; LUNET, N.; PADRÃO, P.; BARROS., H. Tabagismo em Portugal. Arquivos de Medicina, Porto, v. 19, n. 5-6, p. 207 -229, 2005.

GINSBERG, D., HALL, S.M.; ROSINSKI, M. Partner interaction and smoking cessation: a pilot study. Addictive Behaviors, Charleston, v. 16, n. 5, p. 195-202, 1991.

GIOVINO, G. Tobacco control and nicotine dependence. Tobacco Control, Londres, v. 5, p. 1-3, 1996.

GLASSMAN A.H.; COVEY L.S.; STETNER F.; RIVELLI S. Smoking cessation and the course of major depression: a follow-up study. Lancet, Oxford, v. 357, n. 9272, p. 1929-1932, 2001.

HALL, S.; TUNSTALL, C.; RUGG, D.; JONES; R.; BENOWITZ, N. Nicotine gum and behavioural treatment in smoking cessation. Journal of Consulting and Clinical Psychology, Washington, v. 53, n. 2, p. 256-258, 1985. 
HALLSTROM, C.; MCCLURE, N. Anxiety and Depression: Your Questions Answered. Edinburgh: Churchill Livingstone(Eds.), 1998.

Hitsman, B.; Borrelli, B.; McChargue, D.; Spring, B.; Niaura, R. History of Depression an smoking cessation outcome: a metaanalysis, Journal of Consulting and Clinical Psychology, Washington, v. 71, p. 657-663, 2003.

HOMISH, G.; KENNETH L. Spousal influence on smoking behaviours in a US community sample of newly married couples. Social Science $\boldsymbol{\alpha}$ Medicine, Boston, v. 61, número, p. 2557-2567, 2005.

LE FOLL, B.; GEORGE, T.P. Treatment of tobacco dependence: integrating recent progress into practice. Canadian Medical Association J ournal, Toronto, v. 177, n. 11, p. 1373-1380, 2007. LENNOX, A.S. Determinants of outcome in smoking cessation, British J ournal of General Practice, Londres, v. 42, n. 359, p. 247-252, 1992.

LEVENTHAL, H.; CAMERON, L. Behavioral theories and the problem of compliance. Patient Education and Counseling, Filadelfia, v. 10, n. 2, p.117-138, 1987.

LIU, X. Cigarette smoking, life stress and behavioral problems in Chinese adolescents. J ournal of Adolescent Health, S. Francisco, v. 33, n. 3, p. 189-192, 2003.

LOVIBOND, P.; LOVIBOND, S. The structure of negative emotional states: Comparison of the depression anxiety stress scales (DASS) with the Beck Depression and Anxiety Inventories. Behavior Research and Therapy, New Brunswick, v. 33, n. 3, p. 335-343, 1995.

MACHAIN, A. C.; VÉlEZ, N. A.; GARCÍA, F. J .; LUGO, E. K.; GARCÍA, S. C.; MORA, M. E. Relación entre el consumo de tabaco, salud mental y malestares físicos en hombres trabajadores de una empresa textil mexicana. Salud Mental, Santiago de Chile, v. 31, n. 4, p. 291-297, 2008.

MAGID, V.; CRAIG R.; COLDER, L.; STROUD, M.; NICHTER, M. Negative affect, stress and smoking in college students: Unique associations independent of alcohol and marijuana use. Pharmacology Biochemistry and Behavior, La Jolla, v. 90, n. 3, p. 357-362, 2008.

MALBERGIER, A.; OLIVEIRA JR, H.P. Dependência de Tabaco e comorbidade psiquiátrica. Psiquiatria Clínica, S. Paulo, v. 32, n. 5, p. 276-282, 2005.

MANNING, B. K.; CATLEY, D.; HARRIS, K. J.; MAYO, M. S.; AHLUWALIA, J. S. Stress and quitting among African American smokers. J ournal of Behavioral Medicine, Denver, v. 28, n. 4, p. 325-333, 2005.

MARTINET, Y.; BOHADANA, A. O tabagismo. Da prevenção à abstinência. Lisboa: Climepsi Editores, 2003. 
MARTINEZ-ORTEGA, J.; JURADO, D.; GONZALEZ, M.; GURPEGUi, M. Nicotine Dependence, use of ilegal drugs and psychiatric morbidity. Addictive Behaviors, Charleston, v. 31, p. 1722-1729, 2006.

MERMELSTEI N, R.; LICHTENSTEIN, E.; MCINTYRE, K. Partner support and relapse in smoking cessation programs. Journal of Consulting and Clinical Psychology, Washington, v. 51, p. 465-466, 1983.

NUNES, E. Consumo de Tabaco: estratégias de prevenção e controle. Cadernos da Direção Geral de Saúde, Lisboa, 2002.

PAIS-RIBEIRO, J.; HONRADO, A.; LEAL, I. Contribuição para o estudo da adaptação portuguesa das escalas de Depressão Ansiedade Stress de Lovibond e Lovibond. Psychologica, Coimbra, v. 36, p. 235-246, 2004.

PAPERWALLA, K. N.; LEVIN, T. T.; WEINER, J.; SARAVAY, S. M. Smoking and depression. The Medical Clinics of North America, Maryland Heights, v. 88, n. 6, p. 1483-1494, 2004.

PARK, E.; TUDIVER, F.; SCHULTZ, J.; CAMPBELL, T. Does Enhancing Partner Support and Interaction Improve Smoking Cessation? A meta-analysis. Annals of Family Medicine, Ohio, v. 2, n. 2, p. 170174, 2004.

PEREIRA, M.G.; AFONSO, F. Partner Interaction Questionnaire. Versão de I nvestigação. Universidade do Minho, 2006. PEREIRA, M.G.; AFONSO, F. Questionário sociodemográfico. Versão de I nvestigação. Universidade do Minho, 2009.

PESTANA, E.; MENDES, B. Tabagismo: 25 perguntas frequentes em pneumologia. Sociedade Portuguesa de Pneumologia, Lisboa, 1999.

PRECIOSO, J; VISEU, F; VILAÇA, T; HENRIQUES, R; LACERDA, T. Educação para a saúde. Braga: Departamento de Metodologias da Educação. Universidade do Minho, 1991.

PUSCHEL, K; THOMPSON, B; CORONADO, G; RIVERA, S; DIAZ, D; GONZALEZ. Tabaquismo en atención primaria: perfil de fumanteas consultantes, creencias e actitudes de los equipos de salud y oportunidades de intervención. Revista Medica de Chile, Santiago de Chile, n. 134, p. 726-734, 2006.

ROHRBAUGH, M.; SHOHAM, V.; TROST, S.; MURAMOTO, M.; CATE, R.; LEISCHOW, S. Couple Dynamics of Change-Resistant Smoking: Toward a Family Consultation Model. Family Process, Malden, v. 40. n. 1, p. 15-31, 2001.

RONDINA, R.; BOTELHO, C.; GOYAREB, R. A Psicologia do Consumo do Tabaco: uma revisão da literatura. Psicologia Saúde e Doença, Lisboa, v. 3, n. 2, p. 179-189, 2002.

ROSAS M.; BATISTA, F. Desenvolvimento de Estratégias de Intervenção Psicológica para a Cessação Tabágica. Análise Psicológica, Lisboa, v. 1, n. 20, p. 45-56, 2002.

RÜGE, J.; ULBRICHT, S.; SCHUMANN, A.; RUMPF, H.J.; JOHN, U; MEYER, C. Intention to Quit Smoking: Is the Partner's Smoking Status Associated with the Smoker's Intention to Quit? 
I nternational J ournal of Behavioral Medicine, Nova Iorque,v. 15, n. 4, 328-335, 2008.

TRIGO, M. O que provoca realmente a mudança nos fumantees? Algumas reflexões. Revista Portuguesa de Clínica Geral, Lisboa, v. 21, s/n, p. 161-182, 2005.

TSOH, J.Y.; HUMFLEET, J.F.; MUÑOZ, R.F.; REUS, V.I.; HARTZ, D.T.; HALL, S.M. Development of Major depression after treatment for smoking cessation. American Journal of Psychiatry, Arlington, v. 157, n. 3, p. 368-374, 2000.

VAN DIERENDONCK, D.; SCHAUFELI, W.; BUUNK, B.P. Burnout and inequity among human service professionals: A longitudinal study. J ournal of Occupational Health Psychology, Washington, v. 6, n. 1, p. 43-52, 2001.

WEST, R.; HAJEK, P. What happens to anxiety levels on giving up smoking? American J ournal of Psychiatry, Arlington, v. 154, $\mathrm{n}$. 11, p. 1589-1592, 1997.

WORLD DEVELOPMENT REPORT. Making services work for poor people. World Bank, 2003.

WORLD HEALTH ORGANIZATION. Tobacco key facts, WHO, Geneva, 2009.

ZELLARS, K. L.; PERREWE, P. L. Affective Personality and the Content of Emotional Social Support: Coping in Organizations. J ournal of Applied Pyschology, Washington ,v. 86, n. 3, p. 459467, 2001.

\author{
Endereço para correspondência \\ Fernanda Afonso \\ Escola de Psicologia, Universidade do Minho, Braga, Portugal. \\ Campus de Gualtar 4710-057 Braga, Portugal \\ Endereço eletrônico: fernandafonso@gmail.com \\ Maria da Graça Pereira \\ Escola de Psicologia, Universidade do Minho, Braga, Portugal. \\ Campus de Gualtar 4710-057 Braga, Portugal \\ Endereço eletrônico: gracep@psi.uminho.pt
}

Recebido em: 02/12/2011

Reformulado em: 11/03/2013

Aceito para publicação em: 12/03/2013

Acompanhamento do processo editorial: Adriana Benevides Soares

\footnotetext{
Notas

* Doutorada em Psicologia da Saúde

** Professora Associada da Escola de Psicologia, Universidade do Minho
} 